\title{
Dosimetric impact of mixed-energy volumetric modulated arc therapy plans for high-risk prostate cancer
}

\author{
Shyam Pokharel \\ Department of Medical Physics, Premier Oncology, Fort Myers, Florida, USA
}

Received August 02, 2013; Revised August 29, 2013; Accepted August 31, 2013; Published Online August 31, 2013

\section{Original Research}

\begin{abstract}
Purpose: This study investigated the dosimetric impact of mixing low and high energy treatment plans for high prostate cancer treated with volumetric modulated arc therapy (VMAT) technique in the form of RapidArc. Methods: A cohort of 12 prostate cases involving proximal seminal vesicles and lymph nodes was selected for this retrospective study. For each prostate case, the single-energy plans (SEPs) and mixed-energy plans (MEPs) were generated. First, the SEPs were created using 6 mega-voltage (MV) energy for both the primary and boost plans. Second, the MEPs were created using 16 MV energy for the primary plan and $6 \mathrm{MV}$ energy for the boost plan. The primary and boost MEPs used identical beam parameters and same dose optimization values as in the primary and boost SEPs for the corresponding case. The dosimetric parameters from the composite plans (SEPs and MEPs) were evaluated. Results: The dose to the target volume was slightly higher (on average $<1 \%$ ) in the SEPs than in the MEPs. The conformity index (CI) and homogeneity index (HI) values between the SEPs and MEPs were comparable. The dose to rectum and bladder was always higher in the SEPs (average difference up to 3.7\% for the rectum and up to $8.4 \%$ for the bladder) than in the MEPs. The mean dose to femoral heads was higher by about $0.8 \%$ (on average) in the MEPs than in the SEPs. The number of monitor units and integral dose were higher in the SEPs compared to the MEPs by average differences of 9.1\% and 5.5\%, respectively. Conclusion: The preliminary results from this study suggest that use of mixed-energy VMAT plan for high-risk prostate cancer could reduce the integral dose and minimize the dose to rectum and bladder, but for the higher femoral head dose.
\end{abstract}

Keywords: Prostate Cancer; Mixed Energy Plan; VMAT; RapidArc

\section{Introduction}

In external beam radiation therapy, treatment techniques such as 3-dimensional conformal therapy (3DCRT), intensity modulated radiation therapy (IMRT), and volumetric modulated arc therapy (VMAT) are generally used to treat prostate cancer with an objective of delivering conformal dose distributions to the target while minimizing the doses to the normal tissues. Since prostate cancer involves the deep-seated target, the high-energy photon beams are generally used for 3DCRT due to their greater penetrating power. ${ }^{1}$

Corresponding author: Shyam Pokharel, PhD; Premier Oncology, 4571 Colonial Blvd, Unit 100, Fort Myers, FL 33966, USA;

Email: pokharel@livemail.uthscsa.edu

Cite this article as:

Pokharel S. Dosimetric impact of mixed-energy volumetric modulated arc therapy plans for high-risk prostate cancer. Int J

Cancer Ther Oncol 2013;1(1):01011. DOI: 10.14319/ijcto.0101.1
However, the photon beams with energy 10 mega-voltage (MV) or higher also create the secondary neutrons due to interaction between the photons and treatment head of the machine. ${ }^{1}$ Despite high-energy photon having an advantage in penetrating power and skin sparing, use of lower energy (6-10 MV) photon beams have been found to be an effective energy choices for the majority of IMRT prostate cases. ${ }^{1,2}$ Furthermore, several studies demonstrated no clear dosimetric advantages using high-energy photon beams for IMRT prostate cases when compared to the low-energy photon beams. ${ }^{2-8}$

Recently, Park et al. ${ }^{8}$ investigated the effect of changing beam energy according to the penetration depths on the quality of IMRT plans for prostate cancer and made the comparisons between mixed-energy plans (MEPs) and single-energy plans (SEPs) of either low or high energy. In that study ${ }^{8}$, Park et al. showed that mixing energy in an IMRT plan for deep-seated tumors could improve the overall plan quality. However, the dosimetric impact of MEPs for pros- 
tate cancer using VMAT technique remains to be addressed. Thus, we investigated the effect of mixing the low energy (6 $\mathrm{MV})$ and high energy (16 MV) treatment plans for prostate cancer treated with VMAT technique in the form of RapidArc (Varian Medical Systems, Palo Alto, CA, USA). The dosimetric comparisons between SEPs and MEPs were done for 12 prostate cases.

\section{Methods and Materials}

A cohort of 12 prostate cases involving proximal seminal vesicles and lymph nodes was selected for this retrospective study. All 12 cases were treated with RapidArc technique at Premier Oncology, Fort Myers. Florida, USA. The computed tomography (CT) simulation of patients was performed in a supine position on the Phillips Brilliance CT Scanner (Philips Healthcare, Andover, MA, USA), and the CT images were acquired with a $3 \mathrm{~mm}$ spacing. The contouring of prostate, proximal seminal vesicles, lymph nodes, and organs at risk (OARs) (rectum, bladder, and femoral heads) was done on the axial slices of the CT in the Eclipse treatment planning system (TPS), version 11.1 (Varian Medical Systems, Palo Alto, CA, USA). The primary clinical target volume $\left(\mathrm{CTV}_{\mathrm{p}}\right)$ was defined as the prostate, seminal vesicles, and lymph nodes, whereas the boost clinical target volume (CTV $\mathrm{b}$ ) was defined as the prostate only. The primary and boost planning target volume $\left(\mathrm{PTV}_{\mathrm{p}}\right.$ and $\mathrm{PTV}$, respectively) was generated with a margin of $7 \mathrm{~mm}$ around the $\mathrm{CTV}_{\mathrm{p}}$ and $C T \mathrm{~V}_{\mathrm{b}}$, respectively, in all directions except in the posterior direction, where a margin of $0.5 \mathrm{~cm}$ was used.

The RapidArc treatment plans of all 12 cases were generated in the Eclipse TPS using 6 and $16 \mathrm{MV}$ X-ray beams Varian Clinac iX (Varian Medical Systems, Palo Alto, CA, USA). Each treatment plan consisted of primary and boost plan, and the total prescription dose was $81 \mathrm{~Gy}$ with a daily dose of $1.8 \mathrm{~Gy}$ over 45 fractions. Furthermore, the prescription dose to the primary plan was $45 \mathrm{~Gy}$ to the PTV , and the prescription dose to the boost plan was $36 \mathrm{~Gy}$ to the $\mathrm{PTV}$. For each prostate case, the SEPs and MEPs were generated.

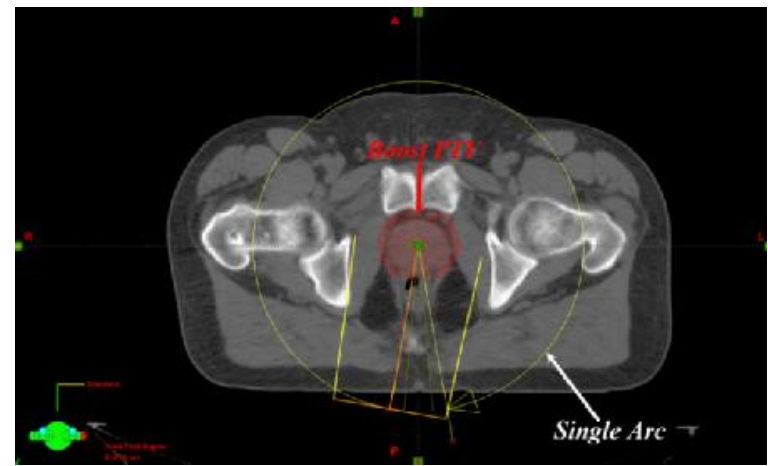

FIG. 1: A transversal view of VMAT (RapidArc) plan set up for boost PTV (case \#7) using one arc in Eclipse treatment planning system. Abbreviations: $\mathrm{VMAT}=$ volumetric modulated arc therapy, $\mathrm{PTV}=$ planning target volume.
First, the SEPs were created using a $6 \mathrm{MV}$ photon beam for both the primary plan and separate boost plan. The treatment plan was set up using one, two or three arcs depending on the size of the target volume. [Figure 1] The length of gantry rotations, collimator angle, and field sizes of the coplanar arcs for the primary as well as boost plans were chosen based on the location of the PTV and OARs using the beam-eye-view (BEV) graphics. [Figure 2]
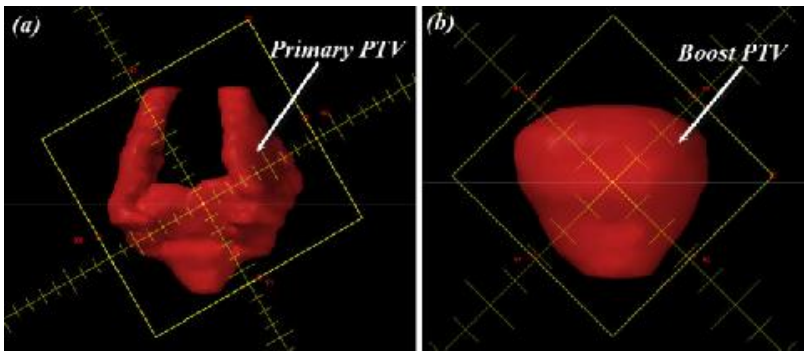

FIG. 2: Beam's-eye-view of case \#7 showing (a) primary planning target volume (PTV), and (b) boost PTV in the Eclipse treatment planning system.

The isocenter of the plan was placed at the center of the target volume (i.e., $\mathrm{PTV}_{\mathrm{p}}$ or $\mathrm{PTV}_{\mathrm{b}}$ ). The primary and boost plans were optimized using Progressive Resolution Optimizer (PRO) (version 11.1). The dose-volume constraints and their weightings were adjusted during the optimization process of SEPs such that at least $95 \%$ of the target volume was covered by the prescription dose while keeping the dose to the OARs as minimum as possible. The plan optimization process was carried out with an objective of meeting the planning criteria listed in Table 1.

TABLE 1: Dose specifications for rectum, bladder, and femoral heads in the composite plan

\begin{tabular}{ccccc}
\hline \hline Organ Limit & $\mathrm{D}_{15 \%}$ & $\mathrm{D}_{25}$ & $\mathrm{D}_{35} \%$ & $\mathrm{D}_{50 \%}$ \\
\hline Rectum & $<75 \mathrm{~Gy}$ & $<70 \mathrm{~Gy}$ & $<65 \mathrm{~Gy}$ & $<60 \mathrm{~Gy}$ \\
Bladder & $<80 \mathrm{~Gy}$ & $<75 \mathrm{~Gy}$ & $<70 \mathrm{~Gy}$ & $<65 \mathrm{~Gy}$ \\
Femoral & Mean Dose $<45 \mathrm{~Gy}$ & & \\
\hline \hline
\end{tabular}

${ }^{*}$ Normal organ limit refers to the volume of that organ that should not exceed the dose limit. Abbreviation: $\mathrm{D}_{\mathrm{x} \%}=$ Dose received by $\mathrm{x} \%$ of total OAR volume, where $\mathrm{x} \%=15,25,35$ and 50; OAR = Organ at risk.

Second, the MEPs were created using a $16 \mathrm{MV}$ photon beam for the primary plan and a $6 \mathrm{MV}$ photon beam for the boost plan. Specifically, the primary MEP used the identical beam parameters and same optimization dose-constraints and their weightings as in the final primary SEP plan for the corresponding case. Similarly, the boost MEP and boost SEP had the same beam parameters and plan optimization values for the corresponding case. No modifications of dose-volume constraints and weightings were made during the optimization processes of MEPs.

The optimized SEPs and MEPs plans were calculated with the anisotropic analytical algorithm (AAA), version 11.1, using dose calculation grid size of $2.5 \mathrm{~mm}$. All calculated 
plans were then normalized such that at least $95 \%$ of the PTV volume was covered by the prescription dose. The primary and boost plans were combined to generate a composite (COMP) plan. This allowed us to perform the dosimetric comparison between the SEPs and MEPs using the dose-volume histograms (DVHs) of the COMP plans that were generated in the Eclipse TPS. The DVH parameters evaluated for the target volume $\left(\mathrm{PTV}_{\mathrm{b}}\right)$ were: mean dose, maximum dose, conformity index (CI) defined as the ratio of volume of the isodose cloud receiving $100 \%$ of the prescription dose $\left(\mathrm{V}_{100 \%}\right)$ to volume of the PTV , and homogeneity index (HI) defined as the ratio of dose at $5 \%$ of the PTV $\left(\mathrm{D}_{5 \%}\right)$ to dose at $95 \%$ of the $\mathrm{PTV}_{\mathrm{b}}$ (D95\%). For rectum and bladder, the volumes that received $70 \mathrm{~Gy}, 40 \mathrm{~Gy}$, and $20 \mathrm{~Gy}$, (V70Gy, V40Gy, and V20Gy, respectively) as well as mean dose were compared. The mean dose to the femoral heads was evaluated. In addition, the number of monitor units (MUs) and normal tissue integral dose were compared too.

For the purpose of comparison, the average percentage difference (Davg.) between the SEPs and MEPs at the corresponding dosimetric parameter of the same case was calculated using Equation 1.

$$
D_{\text {avg. }}(x)=\frac{1}{12} \sum_{n=1}^{12}\left[\frac{(\text { SEP })_{n}-(\text { MEP })_{n}}{(S E P)_{n}} \times 100\right]
$$

where $\boldsymbol{X}$ is a corresponding dosimetric parameter in the COMP SEPs and MEPs for the $\mathrm{n}^{\text {th }}$ case. In Equation 1, the $D_{\text {avg. }}$ is expressed in percentage and averaged over all twelve cases in this study. At a given dosimetric parameter, a posi-

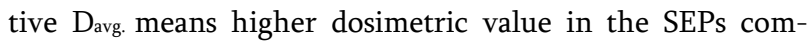
pared with the MEPs, and a negative $D_{\text {avg. means higher }}$ dosimetric value in the MEPs compared with the SEPs. The statistical analysis was done using paired two-sided student's t-test in a Microsoft Excel spreadsheet, and a $p$-value of less than 0.05 was considered to be statistically significant.

\section{Results}

Table 2 and Figures 3, 4, 5, and 6 summarize the dosimetric results in the COMP plans, and the values are averaged over the twelve analyzed cases. The dosimetric results obtained in this study were clinically acceptable.

The maximum and mean doses to the target volume were slightly higher in the SEPs than in the MEPs by an average difference of less than $1 \%$, and the results showed the statistical significance with $p$-values of 0.001 and 0.044 for the maximum and mean dose, respectively. The CI and HI values between SEPs and MEPs were comparable with average differences of $1 \%$ for the CI $(p=0.009)$ and $0.4 \%$ for the HI ( $p=$ $<0.000$ ) showing statistical significance.

The dose to the rectum was always higher in the SEPs and

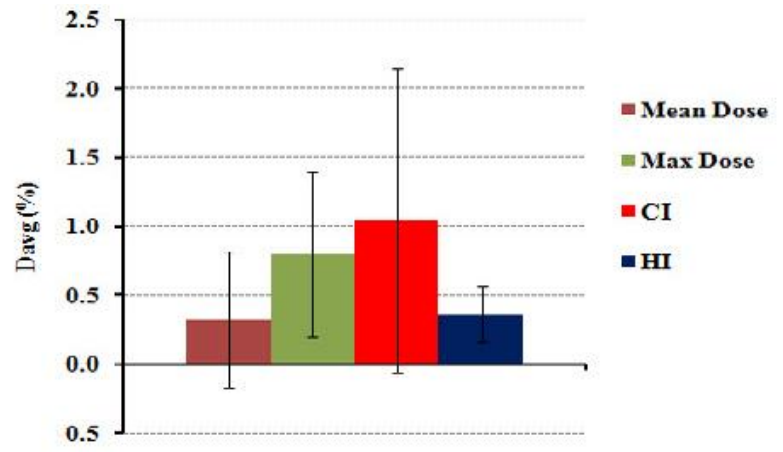

FIG. 3: The Davg. (\%) between SEPs and MEPs for the PTV doses, CI, and HI. The values are averaged over the twelve analyzed cases. Note: The error bars represent the standard deviations. The Davg. (\%) is defined in equation 1 (Materials and Methods). Abbreviations: $D_{\text {avg. }}=$ average difference, SEPs = single energy plans, MEPs = mixed energy plans, PTV = planning target volume, $\mathrm{CI}=$ conformity index, $\mathrm{HI}=$ homogeneity index.

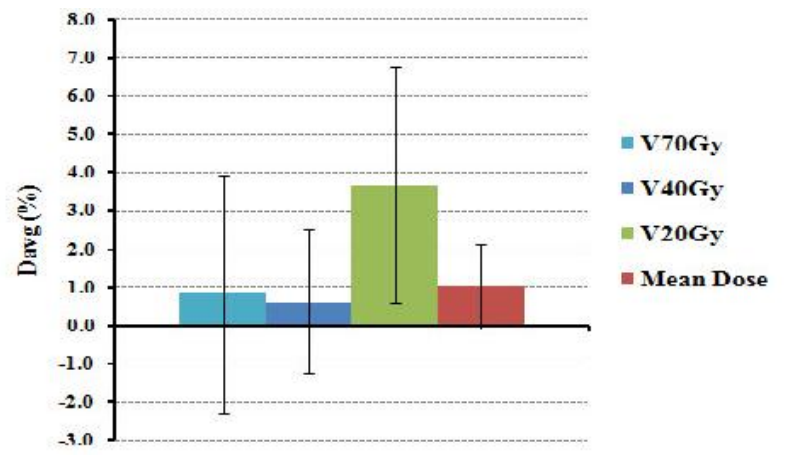

FIG. 4: The Davg. (\%) between SEPs and MEPs for the $\mathrm{V}_{70 G y}, \mathrm{~V}_{40 G y}$, $V_{20 G y}$, and mean does to the rectum. The values are averaged over the twelve analyzed cases. Note: The error bars represent the standard deviations. The Davg. (\%) is defined in Equation 1 (Materials and Methods). Abbreviations: $D_{\text {avg. }}=$ average difference, $\quad$ SEPs = single energy plans, $\mathrm{MEPs}=$ mixed energy plans, $\mathrm{V}_{\mathrm{nGy}}=$ percentage volume irradiated by $n \mathrm{~Gy}$ or more of a certain structure

lower in the MEPs with an average difference ranging from $0.6 \%$ (at $\mathrm{V}_{40 \mathrm{~Gy}}$ ) to $3.7 \%$ (at $\mathrm{V}_{20 \mathrm{~Gy}}$ ). The statistical significance was obtained for the mean dose $(p=0.009)$ and $\mathrm{V}_{20 \mathrm{~Gy}}(p=$ 0.003 ), whereas the statistical significance was not seen for the $\mathrm{V}_{70 \mathrm{~Gy}}(p=0.427)$ and $\mathrm{V}_{40 \mathrm{~Gy}}(p=0.277)$. Similar to the dosimetric results for the rectum, the dose to the bladder was higher in the SEPs and lower in the MEPs. However, the range of average difference values between the SEPs and MEPs were larger for bladder compared to the one for rectum. Specifically, the average difference values in bladder ranged from $0.1 \%$ (at $\mathrm{V}_{20 \mathrm{~Gy}}$ ) to $8.4 \%$ (at $\mathrm{V}_{40 \mathrm{~Gy}}$ ). Furthermore, the statistical significance was obtained for the mean dose $(p$ $<0.000)$, $\mathrm{V}_{70 G \mathrm{y}}(p=0.007)$, and $\mathrm{V}_{40 \mathrm{~Gy}}(p=0.002)$, whereas the results for $\mathrm{V}_{20 \mathrm{~Gy}}(p=0.384)$ were not statistically significant. 
TABLE 2: Comparison of dosimetric parameters for the single and mixed energy composite (primary + boost) plans.

\begin{tabular}{|c|c|c|c|c|}
\hline & & $\begin{array}{c}\text { SEP } \\
(\text { Avg. } \pm \text { SD })\end{array}$ & $\begin{array}{c}\text { MEP } \\
(\text { Avg. } \pm S D)\end{array}$ & $p$-value \\
\hline $\begin{array}{c}\text { PTVb } \\
(127.2 \pm 35.2 \mathrm{cc})\end{array}$ & $\begin{array}{c}\text { Mean Dose }(G y) \\
\text { Max. Dose }(G y) \\
\text { CI } \\
\text { HI }\end{array}$ & $\begin{array}{c}83.2 \pm 0.4 \\
86.3 \pm 0.6 \\
1.09 \pm 0.05 \\
1.03 \pm 0.00\end{array}$ & $\begin{array}{c}82.9 \pm 0.3 \\
85.6 \pm 0.6 \\
1.08 \pm 0.05 \\
1.03 \pm 0.00\end{array}$ & $\begin{array}{c}0.044 \\
0.001 \\
0.009 \\
<0.000 \\
\end{array}$ \\
\hline $\begin{array}{c}\text { Rectum } \\
(77.6 \pm 47.1 \mathrm{cc})\end{array}$ & $\begin{array}{c}\text { Mean Dose (Gy) } \\
\text { V70Gy }(\%) \\
\text { V }_{40 G y}(\%) \\
\text { V20Gy }(\%)\end{array}$ & $\begin{array}{c}34.6 \pm 3.9 \\
6.5 \pm 2.8 \\
26.1 \pm 7.0 \\
89.3 \pm 3.9 \\
\end{array}$ & $\begin{array}{c}34.3 \pm 3.9 \\
6.5 \pm 2.8 \\
25.9 \pm 6.9 \\
86.1 \pm 13.2\end{array}$ & $\begin{array}{l}0.009 \\
0.427 \\
0.277 \\
0.003\end{array}$ \\
\hline $\begin{array}{c}\text { Bladder } \\
(325.9 \pm 218.2 \mathrm{cc})\end{array}$ & $\begin{array}{c}\text { Mean Dose (Gy) } \\
\text { V70Gy }(\%) \\
\text { V }_{40 G y}(\%) \\
\text { V }\end{array}$ & $\begin{array}{c}43.2 \pm 5.2 \\
9.3 \pm 4.3 \\
45.4 \pm 17.2 \\
99.8 \pm 0.5\end{array}$ & $\begin{array}{c}42.2 \pm 5.0 \\
9.0 \pm 4.1 \\
41.4 \pm 15.3 \\
99.7 \pm 0.8\end{array}$ & $\begin{array}{c}<0.000 \\
0.007 \\
0.002 \\
0.384 \\
\end{array}$ \\
\hline $\begin{array}{l}\text { Femoral Heads } \\
(135.7 \pm 16.5 \mathrm{cc})\end{array}$ & Mean Dose (Gy) & $28.0 \pm 3.8$ & $28.2 \pm 3.4$ & 0.684 \\
\hline $\begin{array}{r}\text { Monitor U } \\
\text { Integral Dose }\end{array}$ & $\begin{array}{l}\text { its (MUs) } \\
\left(10^{5} \mathrm{~Gy}-\mathrm{cc}\right)\end{array}$ & $\begin{array}{l}590 \pm 35 \\
3.2 \pm 0.5\end{array}$ & $\begin{array}{l}538 \pm 34 \\
3.0 \pm 0.5\end{array}$ & $\begin{array}{l}<0.000 \\
<0.000\end{array}$ \\
\hline
\end{tabular}

Abbreviations: $\quad \mathrm{SEP}=$ Single Energy Plan, $\mathrm{MEP}=$ Mixed Energy Plan, Avg. $=$ Average, $\mathrm{SD}=$ Standard Deviation, $\mathrm{PTV} \mathrm{b}=$ Boost Planning Target Volume, Max. Dose = Maximum Dose, $\mathrm{V}_{\mathrm{nGy}}=$ Percentage volume irradiated by $\mathrm{n}$ Gy or more of a certain structure, CI = Conformity Index, HI = Homogeneity Index. (The values are averaged over the 12 analyzed cases. The $p$-values were obtained from paired two-sided student's t-test. The p-values less than 0.05 were considered to be statistically significant).

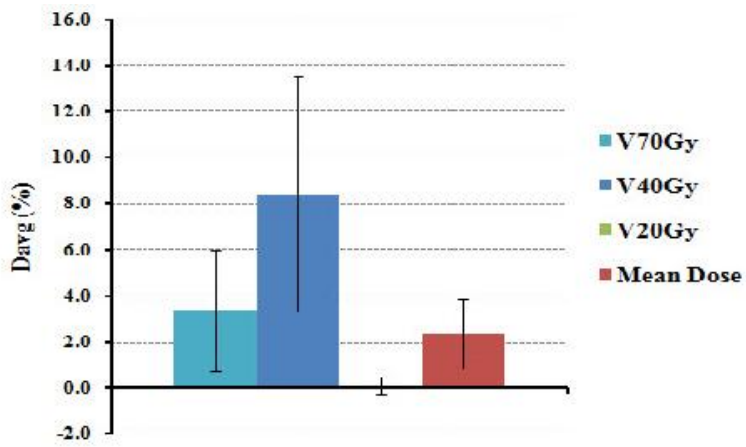

FIG. 5: The Davg. (\%) between SEPs and MEPs for the V70Gy, V40Gy, $\mathrm{V}_{20 \mathrm{~Gy}}$, and mean does to the bladder. The values are averaged over the twelve analyzed cases. Note: The error bars represent the standard deviations. The Davg. (\%) is defined in equation 1 (Materials and Methods). Abbreviations: Davg. = average difference, $\mathrm{SEPs}=$ single energy plans, MEPs = mixed energy plans, $\mathrm{V}_{\mathrm{nGy}}=$ percentage volume irradiated by $n$ Gy or more of a certain structure

In contrast to the results seen for the rectum and bladder in this study, the mean dose to the femoral heads was higher in the MEPs by an average difference of $0.8 \%$ with no statistical significance $(p=0.684)$. In comparison to the MEPs, the number of MUs and integral dose were higher in the SEPs by average differences of $9.1 \%(p<0.000)$ and $5.5 \%(p<0.000)$, respectively, showing the statistical significances.

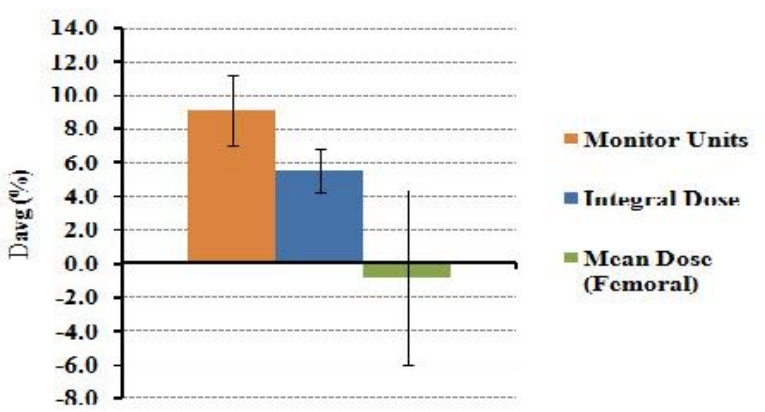

FIG. 6: The Davg. (\%) between SEPs and MEPs for the femoral head mean dose, normal tissue integral dose, and MUs. The values are averaged over the twelve analyzed cases. Note: The error bars represent the standard deviations. The $\mathrm{Davg}_{\text {av. }}(\%)$ is defined in equation 1 (Materials and Methods). Abbreviations: Davg.= average difference, $\mathrm{SEPs}=$ single energy plans, $\mathrm{MEP}=$ mixed energy plans, MUs = Monitor Units.

\section{Discussion}

In this study, we investigated the dosimetric impact of mixing low energy (6 MV) and high energy (16 MV) treatment plans for prostate cancer treated with RapidArc technique. The results from this study showed no clear dosimetric differences between the SEPs and MEPs for the target volume. However, the results suggested that the use of mixed energy treatment plans for prostate cancer could potentially reduce the dose to the OARs, especially for bladder and rectum. 
The use of lower energy photon beams generally minimizes the head leakage, internal scatter, and secondary neutrons. ${ }^{2-7}$ However, the low-energy photon beams also requires greater number of MUs to deposit high doses in the area peripheral to the target, resulting increase in the integral dose and radiation exposure to the OARs. ${ }^{4}$ The results in our study also showed that the number of MUs in the lower energy (6 MV) plans (i.e., SEPs) were about $9 \%$ higher (on average) in comparison to the MEPs that contained higher energy (16 MV) photon beam. Furthermore, the integral dose to the normal tissues was lower in the MEPs by about 5.5\% (on average), and this would also reduce the radiation-induced secondary cancer. ${ }^{9,10}$

The dosimetric differences in the treatment plans from the use of low and high energy photon beams depend on the beam modeling employed within the dose calculation algorithm. ${ }^{11}$ In this study, we used AAA to calculate the dose in all treatment plans. Several studies ${ }^{12-17}$ have documented the limitation of AAA in estimating the dose more accurately when heterogeneous media are involved along the photon beam path. Recently, a number of studies have shown that Acuros XB, new dose calculation algorithm employed within Eclipse TPS, is more accurate than AAA for photon dose calculation, especially in the heterogeneous media. ${ }^{14-17}$ The dosimetric and radiobiological impact of Acuros $\mathrm{XB}$ on the prostate cancer treatment plans due to change in photon beam energy will be an interesting topic for future studies.

\section{Conclusion}

The preliminary results from this study suggest that use of mixed-energy VMAT plan for high-risk prostate cancer could reduce the integral dose and minimize the dose to rectum and bladder, but for the higher femoral head dose.

\section{Competing interests}

The authors declare that they have no competing interests.

\section{References}

1. NCRP. Report No, 79: Neutron contamination from medical electron accelerators. Bethesda, Maryland; NCRP. 1987.

2. Soderstrom S, Eklof A, Brahme A: Aspects on the optimal photon beam energy for radiation therapy. Acta Oncol 1999; 38: 179-187.

3. Pirzkall A, Carol MP, Pickett B, Xia P, Roach M 3rd, Verhey LJ. The effect of beam energy and number of fields on photon-based IMRT for deep-seated targets. Int $J$ Radiat Oncol Biol Phys 2002; 53: 434-442.

4. Subramanian TS. Linear accelerators used for IMRT should be designed as small field, high intensity, intermediate energy units [For the proposition]. Med Phys 2002; 29: 2526-28.

5. Söderstrom S, Eklöf A, Brahme A. Aspects on the optimal photon beam energy for radiation therapy. Acta Oncol 1999; 38: 179-187.

6. Welsh JS, Mackie TR, Limmer JP. High-energy photons in IMRT: uncertainties and risks for questionable gain. Technol Cancer Res Treat 2007; 6: 147-149.

7. Sun M and Ma L. Treatments of exceptionally large prostate cancer patients with low-energy intensity-modulated photons. J Appl Clin Med Phys 2006;7: 43-49.

8. Park JM, Choi CH, Ha SW, Ye SJ. The dosimetric effect of mixed-energy IMRT plans for prostate cancer. J Appl Clin Med Phys 2011;12:3563.

9. Brenner DJ, Curtis RE, Hall EJ, Ron E. Second malignancies in prostate carcinoma patients after radiotherapy compared with surgery. Cancer 2000; 88: 398-406.

10. Hall EJ, Wuu CS. Radiation-induced second cancers: the impact of 3D-CRT and IMRT. Int J Radiat Oncol Biol Phys 2003; 56: 83-88.

11. Madani I, Vanderstraeten B, Bral S, et al. Comparison of $6 \mathrm{MV}$ and $18 \mathrm{MV}$ photons for IMRT treatment of lung cancer. Radiother Oncol 2007; 82: 63-69.

12. Rana SB. Dose prediction accuracy of anisotropic analytical algorithm and pencil beam convolution algorithm beyond high density heterogeneity interface. South Asian J Cancer 2013; 2: 26-30.

13. Robinson D. Inhomogeneity correction and the analytic anisotropic algorithm. J Appl Clin Med Phys 2008; 9: 112-122.

14. Rana S, Rogers K. Dosimetric evaluation of Acuros $\mathrm{XB}$ dose calculation algorithm with measurements in predicting doses beyond different air gap thickness for smaller and larger field sizes. J Med Phys 2013; 38: 9-14.

15. Bush K, Gagne IM, Zavgorodni S, Ansbacher W, Beckham W. Dosimetric validation of Acuros XB with Monte Carlo methods for photon dose calculations. Med Phys 2011; 38: 2208-2221.

16. Han T, Mourtada F, Kisling K, Mikell J, Followill D, Howell R. Experimental validation of deterministic Acuros XB algorithm for IMRT and VMAT dose calculations with the Radiological Physics Center's head and neck phantom. Med Phys 2012; 39: 2193-2202.

17. Rana S, Rogers K, Lee T, Reed D, Biggs C. Verification and dosimetric impact of Acuros XB algorithm for stereotactic body radiation therapy (SBRT) and RapidArc planning for non-small-cell lung cancer (NSCLC) patients. Int J Med Phys Clin Eng Radiat Oncol 2013; 2: 6-14. 